\title{
Comparison of Experimental and Theoretical Characteristics of Linear Waves in the Submerged Air Jet
}

\author{
Linar Gareev ${ }^{1}$, Anastasia Chicherina ${ }^{1}$, Alexander Reshmin ${ }^{1}$, Vladimir Trifonov ${ }^{1}$, \\ Vasily Vedeneev ${ }^{1}$, Julia Zayko ${ }^{1}$ \\ ${ }^{1}$ Institute of Mechanics, Lomonosov Moscow State University \\ 1 Michurinsky prospekt, Moscow, Russia \\ gareev.lr@yandex.ru; chicherina@imec.msu.ru; alexreshmin@ rambler.ru; trifonovvl@mail.ru; \\ vasily.vedeneev@mail.ru; juliazaiko@yandex.ru
}

\begin{abstract}
A submerged air jet of round cross section, $0.12 \mathrm{~m}$ in diameter and with a long laminar region (up to 5 jet diameters) was obtained experimentally at the Reynolds number 5030. Using the linear stability analysis, two branches of growing perturbations were found, which are generated by three inflection points on the experimental jet profiles. Ranges of growing perturbation frequencies were obtained, as well as their growth rates, wavelengths and radial distributions of amplitudes. Experiments, in which controlled perturbations were introduced into the jet with a long laminar region, were conducted. Characteristics of waves, which are growing in experiments, are close to the predictions of the linear stability theory. Consequently, the linear stability theory applied to the submerged jet is validated experimentally.
\end{abstract}

Keywords: linear stability theory, Rayleigh equation, submerged air jet

\section{Introduction}

Evolution of small perturbations, predicted by the linear stability theory, was confirmed experimentally only for several classes of flows [1 - 5]. It is very difficult to organize suck kind of experiments for submerged jets, in which the critical Reynolds numbers $\left(\mathrm{Re}_{\mathrm{cr}}\right)$ of transition to turbulence are very low under normal conditions: in the case of the jet with round cross section $\mathrm{Re}_{\mathrm{cr}}$ lies in the range from 14 to $44[6,7]$. Calculations within the linear stability theory applied to submerged jets were backed up experimentally only for turbulent flows (where transition regions were about $1-2$ diameters). Moreover, in those experiments the introduced disturbances were intensive and had significant influence on the flow $[8,9,10]$.

At the Institute of Mechanics of Lomonosov Moscow State University the facility has been created that generates a submerged jet of $0.12 \mathrm{~m}$ in diameter (D) and with a long laminar region $\sim 5 \mathrm{D}$ at the Reynolds numbers $(\mathrm{Re})$ in the range 5000 $-10000[11,12]$. Such jet provides the possibility to conduct detailed experimental observations of perturbations growth and to compare them with predictions of the linear stability theory applied to velocity profiles at different distances from the orifice. In this paper, it is shown that experimentally obtained ranges of frequencies, wave-lengths, growth rates, radial distributions of amplitudes for the most growing disturbance are in accordance with the predictions of linear theory.

\section{The Jet with a Long Laminar Region}

In $[11,12]$ the jet with a long laminar region and the facility forming it are described thoroughly. Hereinafter, the theoretical analysis and experiments are conducted for the flow with $\mathrm{Re}=5030$, based on the diameter $\mathrm{D}$ and average velocity (the axis speed $U_{c}=1.5 \mathrm{~m} / \mathrm{s}$ ). The velocity profiles at different distances from the orifice were measured by a thermoanemometer, then compared with profiles obtained from stationary laminar calculation of the jet (see [12]), and finally approximated by analytical functions (Fig. 1). 


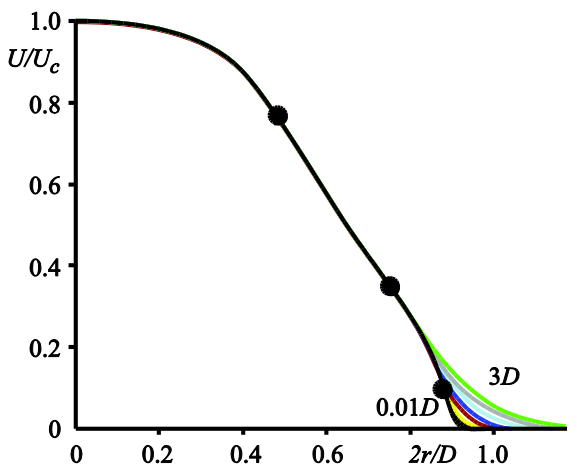

Fig. 1: Velocity profiles at different distances from the orifice nondimensionalised by a maximum speed and radius of the jet (generalized inflection points of the initial profile are denoted by circles).

\section{Theoretical Stability Analysis of the Jet}

Theoretical linear stability analysis is carried out in inviscid approximation (using the Rayleigh equation). An inviscid approach is motivated by comparatively high Reynolds number of the flow under consideration. The axisymmetric mode is studied. Nine profiles of longitudinal velocity at different distances from the orifice are analyzed. At the distances along the jet axis up to $1.5 \mathrm{D}$ there are three generalized inflection points (points where $\left(\boldsymbol{u}^{\prime}(\boldsymbol{r}) / \boldsymbol{r}\right)^{\prime}=\mathbf{0}$, see Fig. 1) at the velocity profiles. It is found that in the considered flow two branches of growing perturbations exist. Speeds of special growth and wavelengths of growing perturbations are obtained. It is shown that the fastest growing perturbations of the first branch have frequencies in the range of $\sim 4 . .8 \mathrm{~Hz}$. Moving downstream, the outer inflection point moves inside the jet, which is caused by jet profile transformation. The transformation occurs as the result of viscous "diffusion" accompanied by the decrease of maximum rate and the reduction of frequency range of growing perturbations. Frequencies of the fastest growing perturbations of the second branch lie in the range of $\sim 4 . .8$ either. Throughout the laminar region, this mode is not affected considerably by the evolution of the jet profile.

\section{Experiment on Introduction of Controlled Perturbations to the Jet}

The facility introducing perturbations to the jet is shown in Fig. 2. Two metal thin strings are pulled on two drivers and on these strings a metal ring of a thicker wire is fixed coaxially with the jet (the section area of strings and ring is chosen in order to prevent the Karman vortex streets formation). The strings and the ring are actuated by the driver, which is capable of set-ting different frequencies and amplitudes, and so the ring follows its motion. Two rings of different diameters are used. The ring \#1 has a diameter lying between two inflection points, which generate the first branch of growing perturbations. The radius of ring \#2 is chosen to be equal to the distance between the jet axis and the inflection point, which generates the second branch of growing perturbations.
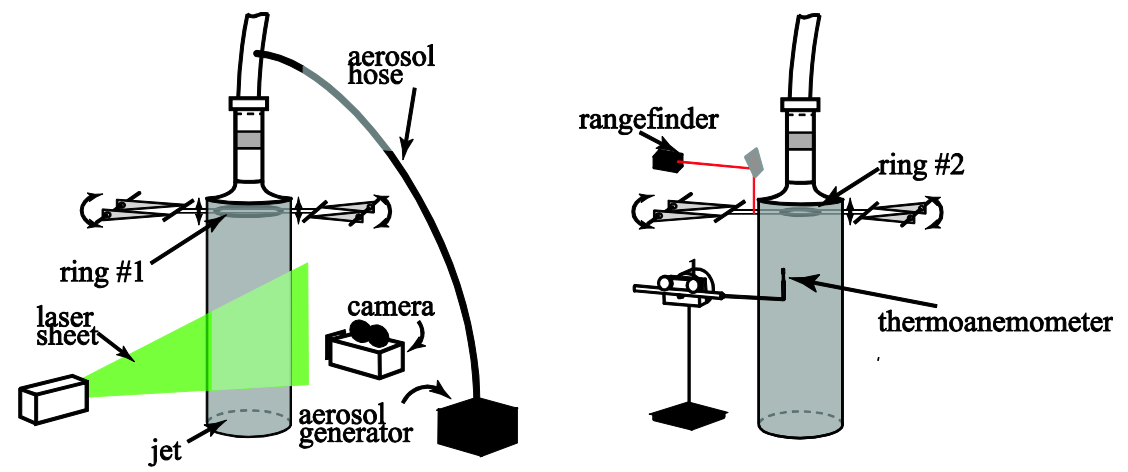

Fig. 2: The scheme of the flow, introduction of perturbations to the jet, laser sheet, thermoanemometer and rangefinder. 
The facility at rest showed no significant effect to the flow as the length of laminar region remained unchanged (Fig.3). Two series of experiments were conducted. The first one - to excite the theoretically obtained first mode (using ring \#1), the second one - to excite the second mode, generated by the inflection point close to the jet axis (using ring \#2). The flow in the first series of experiments was visualized with small glycerin particles and the filming was made in the section laser sheet with ring \#1 oscillating in the range from 0 to $12 \mathrm{~Hz}$ with the step of $0.25 \mathrm{~Hz}$ (see the photos of jets in Fig. 3). The pictures of the jet under different frequencies of oscillation and lengths of waves occurring in the jet boundary were obtained.

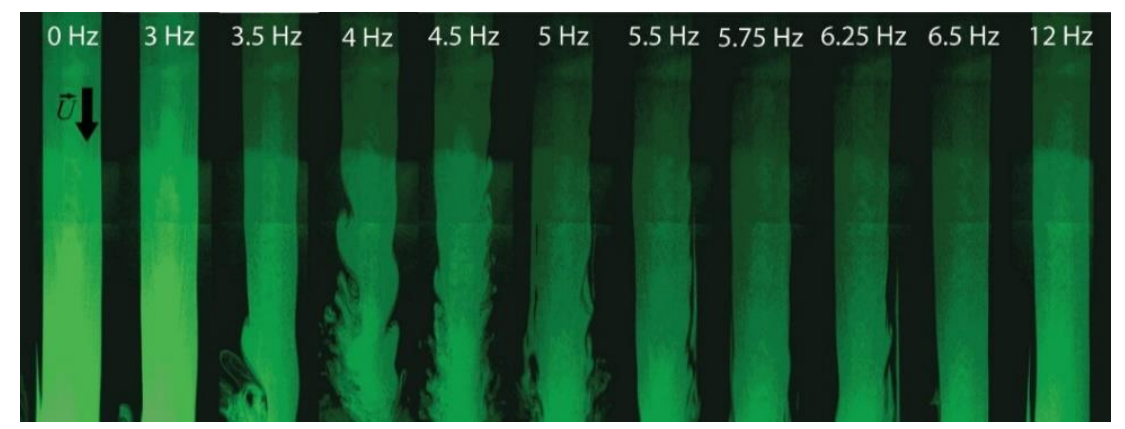

Fig. 3: Photographs of the jet in the laser sheet section under different frequency oscillation of ring \#1.

In the second series of experiments, phase-locked recording of the thermoanemometer and rangefinder signals was made. The thermoanemometer measured velocity and velocity fluctuations along the jet radius at different distances from the start of the jet. Correlation maps of recorded signals were made for different frequencies of ring oscillations (Fig. 4). From these pictures wavelengths of disturbances with the given frequency were extracted.
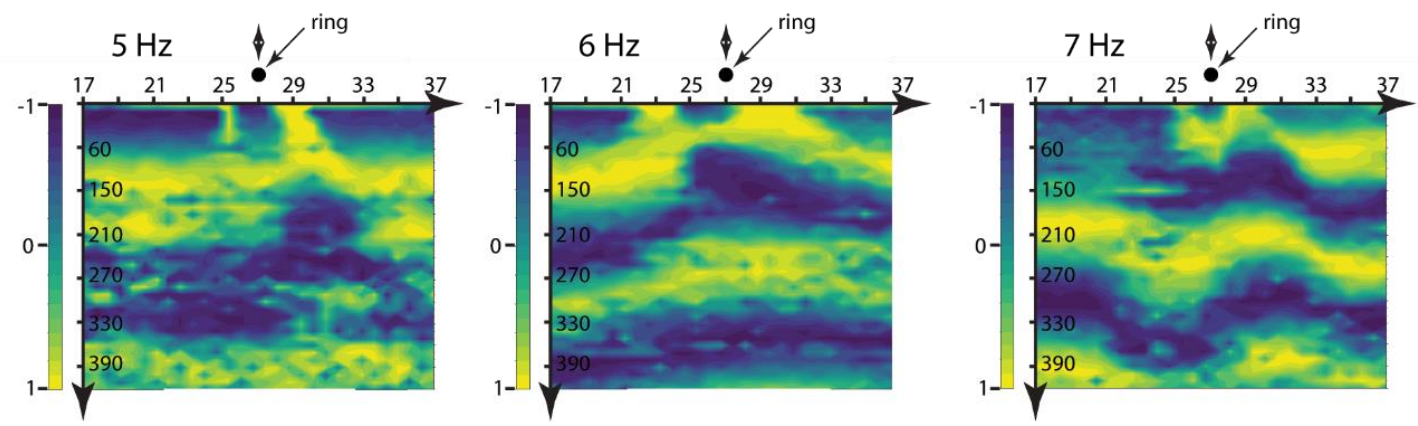

Fig. 4: Correlation maps of phase-locked thermoanemometer and rangefinder signals for different frequencies of ring \#2 oscillations.

\section{Comparison of Experimental Data with Linear Stability Theory Predictions}

The jet in the laser sheet section under different oscillation frequencies of ring \#1 (and fixed oscillation speed) is shown in Fig. 3. It is obvious that at frequencies higher than $6.5 \mathrm{~Hz}$ and lower than $3 \mathrm{~Hz}$ there is no significant effect of oscillations on the jet. In the range of $3.25-6.25 \mathrm{~Hz}$ the decrease of the laminar region occurs. These frequencies correspond to the range of the most growing perturbations obtained theoretically. Wavelengths of perturbations arising at the jet boundary were derived from filming. There is a good accordance between them and those, obtained theoretically (see the comparison in Fig. 5a).

Wavelengths obtained from the correlation pictures under different oscillation frequencies of ring \#2 are in excellent agreement with wavelengths of the second branch of growing perturbations (Fig. 5a, triangle symbols).

Theoretical and experimental radial distributions of axial velocity perturbation amplitudes are also in agreement (Fig. $5 b)$, except for a small region at the jet boundary, where the plane-parallel assumption of the linear stability theory is not satisfied. 

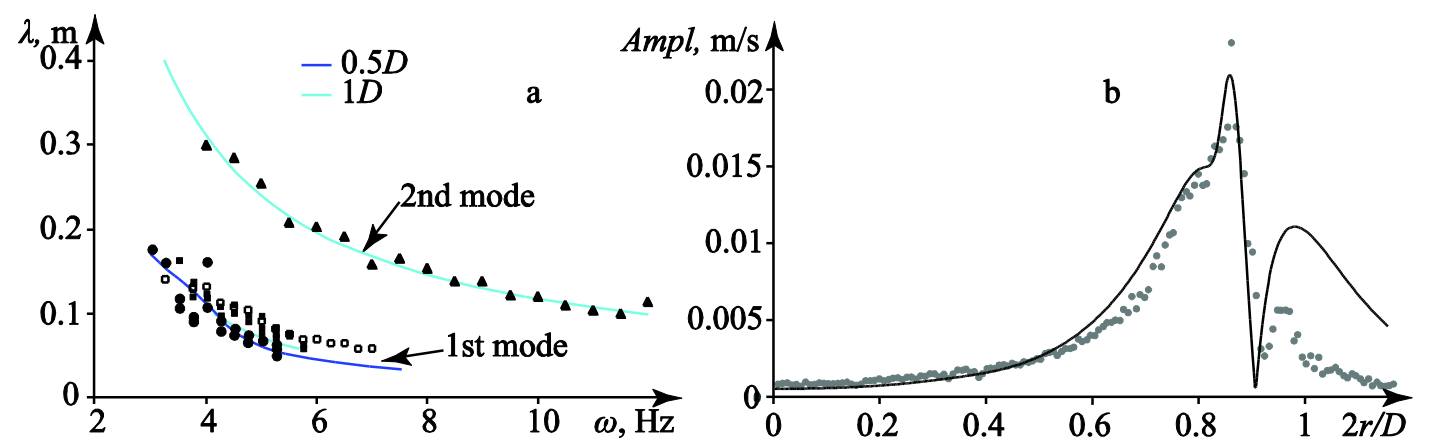

Fig. 5: (a) Comparison of experimental and theoretical wavelengths $\lambda$ versus excitation frequencies $\omega$. Experimental wavelengths are denoted by symbols, theoretical - by curves. (b) Comparison of theoretical (black curve) and experimental (grey points) radial distributions of axial velocity perturbation amplitudes. Ring oscillation frequency is $5 \mathrm{~Hz}$, distance from the orifice is $0.5 \mathrm{D}$.

\section{Conclusion}

In this study, it is shown experimentally that linear inviscid stability theory predicts accurately wave properties of a laminar submerged jet of round cross section at the Reynolds numbers $\sim 5000$. In experiments, according to theoretically obtained eigenmodes of two branches of growing perturbations, both types of waves were detected. Experimentally found wavelengths, frequency ranges and radial distributions of axial velocity perturbation amplitudes of growing waves are close to those theoretically predicted under the assumptions of linear inviscid stability theory.

\section{Acknowledgements}

This work is supported by the Russian Science Foundation grant 20-19-00404.

\section{References}

[1] G.B. Schubauer and H. K. Skramstad, "Laminar boundary-layer oscillations and transition on a Flat plate," J. Res. Nat. Bur. Stand., vol. 38, pp. 251-292, 1947.

[2] A.V. Boiko, K.J.A. Westin, B.G.B. Klingmann, V.V. Kozlov, P.H. Alfredsson, "Experiments in a boundary layer subjected to free stream turbulence. Part 2. The role of TS-waves in the transition process," J. Fluid Mech., vol.281, pp. 219-245, 1994.

[3] M. Nishioka, S. Iida, Y. Ichikawa, "An experimental investigation of the stability of plane Poiseuille flow," J. Fluid Mech., vol. 72(4), pp. 731-751, 1975.

[4] V.V. Kozlov, S.P. Ramazanov, "Experimental investigation of Poiseuille flow stability," Izv. Sib. Otd. Akad. Nauk SSSR, Ser. Tekh. Nauk, vol. 8, pp. 45-48, 1981 (In Russian).

[5] W. Pfenniger, "Transition in the inlet length of tubes at high Reynolds numbers," in Boundary Layer and Flow Control, G.V. Lachman, Ed. New York: Pergamon Press, 1961, pp.970-980.

[6] P.J. Morris, "The spatial viscous instability of axisymmetric jets," J. Fluid Mech., vol. 77(3), pp. 511-526, 1976.

[7] V. Shtern and F. Hussain, "Effect of deceleration on jet instability," J. Fluid Mech., vol. 480, pp. 283-309, 2003.

[8] S.C. Crow and F.H. Champagne, "Orderly structure of jet turbulence," J. Fluid Mech., vol. 48, pp. 547-591, 1971.

[9] J. Cohen and I. Wygnanski, "The evolution of instabilities in the axisymmetric jet. Part 1. The linear growth of disturbances near the nozzle," J. Fluid Mech., vol.176, pp. 191-219, 1987.

[10] R.A. Petersen, M. M. Samet, "On the preffered mode of jet instability," J. Fluid Mech., vol. 194, pp. 153-173, 1988.

[11] J.S. Zayko, A.I. Reshmin, S.Kh. Teplovodskii, A.D. Chicherina, "Investigation of submerged jets with amplified length of initial laminar region," Fluid Dynamics, vol. 1, pp. 97-106, 2018.

[12] J. Zayko, S. Teplovodskii, A. Chicherina, V. Vedeneev, A. Reshmin, "Formation of free round jets with long laminar regions at large Reynolds numbers," Phys. Fluids., vol. 30(043603), 2018. 